\title{
Enzo Traverso, La historia como campo de batalla. Interpretar las violencias del siglo XX, México, FCE, 2016.
}

Un pasado obsesiona a este presente, escribiría Michel de Certeau en La escritura de la historia (2010, p. 38), pero no es ya el de los fantasmas que callan en el silencio sepulcral de la muerte, sino el de los síntomas de una escritura patologizada por la ausencia aun latente del tiempoque-ya-no-es. Ese pasado es el de la fragmentación de las creencias, la búsqueda esquizoica por reinsertar lo sagrado en lo Real, del desgarramiento de tejidos sociales por la imposibilidad de enunciar al otro. Si el siglo XVIII fue marcado por los grandes proyectos civilizatorios y en XIX asistimos a la consolidación de los Estados con sus instrumentos institucionales y espíritus nacionales.

El siglo pasado contempló cómo el gesto (la muerte) fue desplazado por su reflejo (la violencia) en la calamitosa precipitación de acontecimientos, esto es, de momentos que estallan en el orden de la relación, teniendo en el trasfondo el cruce que le impregna su condición de arbitrariedad y azar cuando los estratos temporales estallan, se hace añicos el sentido y las coherencias recibidas se desvanecen en el aire. Pero fue también la época de la emergencia revolucionaria con las experiencias mexicana, bolchevique, china, turca, cubana, nicaragüense, en fin, incluso el episodio fascista en Italia era adjetivado por la prensa como "Gran Revolución”, pero aun cuando este sueño pronto descarnó las pesadillas del totalitarismo, permite entrever formas de resistencia y prácticas de humanidad de forma paralela a los peligros del desencantamiento agudizado y el odio exacerbado indispensables hoy por hoy.

La historia como campo de batalla, publicado originalmente en Francia en 2011, es reeditado en México para actualizar la agenda de trabajo en lengua hispana de los estudiosos 
del pasado. El título revela las intenciones de su autor inmediatamente. Por una parte, ofrecer a los lectores recursos innovadores a partir del potencial epistemológico de los aportes de tan diversos intelectuales como Reinhart Koselleck, Michel Foucault, Giorgio Agamben, Dominick LaCapra, Frederic Jameson, Perry Anderson, Jürgen Habermas o Toni Negri. Por otra, interpretar las violencias que desgarraron el siglo XX y van desde revoluciones utópicas, guerras totales, gobiernos totalitarios y contrarrevoluciones hasta genocidios, dispositivos biopolíticos sobre los cuerpos y las poblaciones, además del proceso de trasformación de la naturaleza del gulag, de Auschwitz o de Hiroshima en objetos de análisis histórica.

En un primer movimiento, Traverso se sitúa como historiador en un contexto específico: el alba de un nuevo Sattelzeit, un tiempo bisagra en el que las palabras transmutan su significado y se dota con un nuevo sentido al relato histórico. Así como Koselleck estaba seguro de haber visto esa emergencia en el periodo que va de la crisis del Antiguo Régimen a la Restauración, Traverso vislumbra un segundo ocaso entre el final de la Guerra de Vietnam y el atentado a las Torres Gemelas. Con base en este argumento se asevera que vivimos inmersos en el desencantamiento del mundo, en el presentismo arrancado de su pasado traumático e incapaz de ver al futuro por la carga de incertidumbre que este posee, imposible de pensarse por fuera del léxico empresarial que lo subsume en rankings, marketing, competencias y coaching.

\footnotetext{
${ }^{1}$ Para Traverso es fundamental ser conscientes de que los temas que él trata han tenido un proceso de historización. El caso de Hiroshima es emblemático: mientras que Le Monde saludó el hongo atómico como una revolución científica y la sociedad mundial aisló a los hibakusha, es decir, los irradiados, como portadores de una enfermedad vergonzosa y les rodeó de un silencio púdico, ya en 1956 Günter Anders capturaba en $L a$ obsolescencia del hombre la novedad absoluta de la bomba atómica como síntoma de "una mutación antropológica aterradora: el advenimiento de una supremacía de la técnica sobre los hombres” (p. 254). Similar efecto está teniendo la decisión de Trump de anunciar la salida de los Estados Unidos del Acuerdo de París al reverdecer la polémica sobre el calentamiento global y la necesidad de una historia ambiental que se nutra de la historia oral y la del tiempo presente, tal como la proponen reconocidos historiadores y psicoanalistas en el compendio Trauma, cultura e historia: Reflexiones interdisciplinarias para el nuevo milenio, coordinado por Francisco A. Ortega Martínez, Bogotá, CES-Universidad Nacional de Colombia.
} 
Apropiándose de los esquemas metodológicos propuestos por Arno J. Mayer, Traverso apunta un crisol de prácticas que se realizan desde este horizonte "post-fin de la historia”. El campo de interés del historiador se ha centrado en el presente, en historizar los contextos que lo rodean y constituyen la base de su observación.

Los grandes puntos de inflexión histórica que desgarraron el siglo XX rehabilitaron el acontecimiento con su autonomía y espesor, con su irreductibilidad a cualquier causalidad determinista, pues en función de su terrible singularidad dejaron de caber en las estructuras al mismo tiempo que las experiencias personales de las víctimas y los subalternos dejaron de pertenecer a una memoria colectivizable debido a su inenarrabilidad. Con ello aparecieron una serie de signos que anunciaban el encuentro de "las napas freáticas de la memoria colectiva” con "el centelleo simbólico del acontecimiento histórico” (Traverso, 2016, p. 18) Traverso recuenta las obras que canonizaron una historiografía de la memoria que no ha cesado su reproducción a partir de Levi, Nora, Yerushalmi y Halbwachs.

A lo largo de un libro, Traverso nos invita a tomar parte de los debates que entabla con ciertos historiadores desde una estrategia retórica que los hace confrontarse conellos, como es el caso de Ian Kershov para contravenir a Götz Ally acerca de los límites interpretativos del “poder pastoral” para explicar el Holocausto y la participación de la sociedad alemana, a Aimé Césaire con su representación del nazismo como una reproducción en pequeña escala de la violencia colonial para contrapuntear la definición de Primo Levi del Holocausto como un unicum de la historia, a Saul Friedländer para evidenciar las debilidades de la Altagsgeschichte de Martin Broszat, o a George Mosse, Emilio Gentile y Zeev Sternhell para contravenir a Jacob Talmon y François Furet cuando perciben en el fascismo y en el comunismo dos gemelos totalitarios gestados en la experiencia jacobina de 1789 . 
Los años no han erosionado las lecciones sistematizadas por Roger Chartier cuando advertía que la escritura histórica del nuevo siglo, en tanto mediación cultural reproducida a través de la comunicación, localiza sus presupuestos ya no en las estructuras o en las mentalidades, sino en las mediaciones, las prácticas y las representaciones, demandando una rigurosidad de primer orden en el tratamiento conceptual que el historiador hace del pasado. En Traverso, como en otros historiadores que sacan provecho de la historia conceptual y de la historia de los lenguajes políticos inspirados por Skinner, Pocock, Koselleck o Lovejoy, esta preocupación es otro de los ejes transversales del texto.

A manera de ejemplo, el fascismo, tan discutido y vaciado de contenido en los debates contemporáneos, debe ser entendido en los términos no de una revolución conservadora ni de una anti-revolución, sino de una contrarrevolución en tanto síntesis de elementos preexistentes y discordantes caracterizados en este libro como modernismo reaccionario, dado que el parentesco de sus distintos elementos constitutivos como movimiento social y en cuanto régimen político fue el anticomunismo, mismo que constituyó el zócalo común de la nebulosa de fascismos europeos bálticos, italiano, alemán, francés e hispanolusitano, cada uno con su recorrido diferente. El espíritu anticomunista no hizo sino potenciarse con la matriz generadora de la Gran Guerra, que sintetizó esa genealogía intelectual al reunir a los ideólogos de la extrema izquierda y la derecha tradicional, al tiempo que generó las condiciones sociales propicias para la movilización de las masas y sus voluntades. En este concierto, Traverso se muestra contrario al recurso de tipos ideales a la manera de Weber en la historia, dado que éste ha generado abusos y desviaciones como en el caso del totalitarismo que en tanto "modelo abstracto [...] se corresponde más con las fantasías literarias de George Orwell que con el funcionamiento real de los regímenes fascistas o comunistas” (Traverso, 2016, p. 202). 
No obstante, uno de los elementos de mayor riqueza epistemológica se manifiesta en una premisa que nos apareja con las cavilaciones vertidas por Lucien Febvre la navidad de 1952 en Combates por la historia: el historiador es emplazado a un constante examen de conciencia, un volver sobre sí, que no hace sino develar que la única historia que existe es la investigación científica del hombre pero no como ente abstracto y eterno idéntico a sí mismo, sino como "hombres comprendidos en el marco de las sociedades de que son miembros". Un repliegue más allá de toda metodología que sólo tiene cabida en el orden de la teoría autorreflexiva desde la cual se pone en acto la virtud cardinal del historiador, el sentido del movimiento, de su propia historicidad y las implicaciones éticas que ello supone.

Lejos han quedado los tiempos de los manuales de Historia repletos de pasos a seguir para la erudición del marco teórico, notas sobre la elaboración del aparato crítico y ejemplos del método documental a la manera de Langloise, Julio Aróstegui, Pierre Vilar o Ernesto de la Torre Villar. Sin embargo, no ha desaparecido el ahínco de integrar parámetros útiles en el ejercicio de las pesquisas del historiador. Así, Traverso plantea un conjunto de reglas, no leyes, que deben regir toda operación historiográfica que se precie sensible a las fluctuaciones de su tiempo: situar un acontecimiento o una idea en su marco epocal y contexto social; historizar la realidad que nos rodea, y abordar los hechos y las ideas desde una perspectiva diacrónica que capte sus mutaciones en la duración; comparar los fenómenos estudiados con otros inscritos en distintas coordenadas temporales y espaciales para tratar de comprender su especificidad; y, finalmente, la precisión al momento de articular la conceptualización antes mencionada con el modo narrativo de la escritura que pone en acto a la historia.

Cuando se discute acerca de la "revolución industriosa” de Jean de Vries, la “terapéutica del exterminio” en Nolte, el carácter autobiográfico de toda producción histórica, el recurso a la memoria personal y el itinerario intelectual en varios historiadores de los 
genocidios y las diásporas, o la propuesta de una "hermenéutica de la mirada" en la historiografía como una posibilidad dada por la condición del desplazamiento que vuelve a los exiliados un sismógrafo sensible y precoz a los movimientos telúricos dada su condición de marginalidad, "el terreno fecundo del escritor que desde entonces ya no puede 'habitar' su propia lengua” (Traverso, 2016, p. 256) como expresa pesarosamente Adorno, Traverso nos provoca a relocalizar la implicación subjetiva del historiador, misma que había sido reprimida por la referencia ritual a un presunto deber deontológico de objetividad empirista que ha quedado descubierta, a la luz de la historikerstreit, como herramienta de legitimación de un orden político dado.

La distancia que el historiador adopta en tanto outsider, según la fórmula de Edward Said, no tiene como objetivo una pretendida neutralidad, sino recabar pruebas para ofrecer una verdad contingente acerca de la interpretación del pasado. Con toda su complejidad y riqueza, el trabajo de los historiadores "está puesto al servicio de la sociedad que lo usa como quiere” en un mundo regido por infraestructuras virtuales, espacios omnipresentes de opinión pública y fiebres nostálgicas de patrimonialización ${ }^{2}$ y humanismos compasivos. No puedo evitar pensar a partir de este libro que el historiador debe reconocerse como un combatiente, en palabras de Eugenia Meyer, un partidario por "el reconocimiento [del] pluralismo [que] puede abrir provechosos espacios de reconocimiento, más allá de identidades nacionales petrificadas” (Traverso, 2016, p. 315).

En resumen, la obra aquí reseñada es doblemente enriquecedora por su propuesta epistemológica y por su potencial pedagógico, por la polifonía historiográfica que la compone y los trazos metodológicos que la entretejen. Es un libro que se apropia de la "melancolía de

\footnotetext{
${ }^{2}$ La reactivación del pasado y el resurgimiento de la memoria como discurso en los espacios públicos han dado forma a "el culto a los lugares de memoria como monumentos fetichizados de una identidad nacional perdida o amenazada” (Traverso, 2016, p. 295). 
izquierda" como motor de la historia y forma critica del discurso normativo actual, que presenta el régimen neoliberal y la economía de mercado como el orden natural del mundo, una exploración empática que ofrece a nuestra mirada un campo de ruinas como acto productor de conocimiento. En nuestro mundo, y particularmente en regiones desgarradas por dictaduras y regímenes autoritarios dedicados al silenciamiento de las voces disidentes, a la normalización de prácticas racistas y clasistas, así como a la ahistorización de las identidades culturales y sexo-genéricas, es un imperativo que el historiador rescate en el pasado formas de resistencia y de sociabilidad alternativas, como nos lo demuestra Traverso: "el siglo XX ha sido la era de la violencia, las guerras totales, los fascismos, los totalitarismos y los genocidios, pero también la era de las revoluciones que naufragaron y de las utopías que se desmoronaron” (Traverso, 2016, p. 324).

Octavio Spíndola Zago Benemérita Universidad Autónoma de Puebla octavio_spindola@hotmail.com 